\title{
Prevalence and determinants of congenital cytomegalovirus infection at a rural South African central hospital in the Eastern Cape
}

\author{
Donald Tshabalala ${ }^{a}$, Howard Newman ${ }^{b *}$, Charles Businge $^{c}$, Sikhumbuzo A Mabunda ${ }^{d}$ iD, Waldette Kemp and Pakama Beja ${ }^{\mathrm{e}}$ \\ ${ }^{a}$ Department of Paediatrics, Nelson Mandela Academic Hospital and Walter Sisulu University, Mthatha, South Africa \\ ${ }^{b}$ National Health Laboratory Service, Port Elizabeth, South Africa and Department of Pathology, Division of Medical Virology, Stellenbosch \\ University, Cape Town, South Africa \\ 'Department of Obstetrics and Gynaecology, Nelson Mandela Academic Hospital and Walter Sisulu University, Mthatha, South Africa \\ ${ }^{d}$ Department of Public Health, Walter Sisulu University, Mthatha, South Africa \\ eNational Health Laboratory Service, Port Elizabeth, South Africa \\ *Corresponding author, email: howard.newman@nhls.ac.za

Background: The Nelson Mandela Academic Hospital (NMAH) in Mthatha, Eastern Cape, is a rural central hospital, serving one of the poorest districts in South Africa. The prevalence of and risk factors for congenital cytomegalovirus (CMV) in this area are not known. The aim was to evaluate the prevalence of congenital CMV and associated risk factors for babies born at NMAH.

Methods: This was a cross-sectional study to determine the prevalence of congenital CMV infection among babies born at Nelson Mandela Academic Hospital. Mother-baby pairs delivered consecutively from Monday to Friday, who gave informed consent, were included. Demographic information was collected on a questionnaire. All babies were tested for congenital CMV using a saliva swab PCR within the first week of life.

Results: A total of 302 births were assessed. Congenital CMV was prevalent in 18 births (5.96\%; 95\% $\mathrm{Cl} 3.29-8.63)$ and had an equal prevalence between HIV-exposed and HIV-unexposed newborns (prevalence ratio [PR] $=1.00 ; 95 \% \mathrm{Cl} 0.94-1.06 ; p=0.869$ ). Conclusions: The prevalence of congenital CMV of 5.96\% is similar to findings from other resource-limited settings. There was no significant association between maternal HIV status and congenital CMV. With the prevalence of congenital CMV being as high as it is in the studied setting, clinicians are advised to have a high index of suspicion, especially when mothers are CMV seropositive.

Keywords: congenital CMV, Mthatha, prevalence, risk factors, South Africa

\begin{abstract}
Introduction
Human cytomegalovirus (CMV) is an enveloped double-stranded DNA virus belonging to the Herpesviridae family of viruses. CMV is distributed worldwide and affects humans of all ages and socio-economic backgrounds, ${ }^{1}$ with congenital CMV being a leading cause of congenital infections worldwide. In resourcelimited settings with very high CMV seroimmunity, congenital CMV rates of $1 \%-5 \%$ have been reported compared with rates of under $1 \%$ in industrialised nations. ${ }^{2}$
\end{abstract}

Maternal CMV infection during pregnancy most often results from close contact with young children. ${ }^{3}$ The risk of vertical transmission to the foetus is far higher with primary maternal infection than with recurrent infection (approximately 30\% versus $1.5 \%) .{ }^{4}$ Advanced gestation and younger primigravid women appear to be factors linked to increased risk of transmission to the foetus. ${ }^{4,5}$

Infants infected as a consequence of primary maternal infection are more likely to have symptoms at birth and suffer long-term sequelae than those infected as a result of maternal recurrent CMV infection. The risk of hearing loss, however, appears to be similar in both groups. ${ }^{6-8}$

Approximately $90 \%$ of neonates with congenital CMV infection will be asymptomatic at birth, with the remaining $10 \%$ having clinically apparent or symptomatic infection, where the disease manifestations can range from mild non-specific findings to multiple organ system involvement. The most commonly observed physical findings are petechial rash, jaundice and hepatosplenomegaly with neurological abnormalities such as microcephaly and lethargy. ${ }^{9}$ Neonates who are asymptomatic at birth have a $10 \%$ chance of developing sensorineural hearing loss later in life. ${ }^{9}$

The diagnosis of congenital CMV relies on virus detection by culture-based methods or PCR on saliva or urine specimens, collected within the first two weeks of life. ${ }^{10}$ After this time period, virological testing cannot discriminate between intrauterine infection and postnatal infection.

An increased frequency of in utero CMV transmission in HIVinfected mothers has been consistently documented in Western countries. ${ }^{2}$ HIV-infected newborns are also more likely to acquire congenital CMV than HIV-uninfected neonates. ${ }^{11}$ In the era of combination antiretroviral therapy (cART), the incidence of vertical transmission of CMV in HIV-positive mothers is falling and is associated with improvements in CD4 count. ${ }^{11}$ Some studies, however, have found no significant decrease in the prevalence of congenital CMV infection in children of HIVinfected mothers receiving prenatal antiretroviral therapy. ${ }^{12}$

The Nelson Mandela Academic Hospital (NMAH) in Mthatha, Eastern Cape, is a rural central hospital, serving one of the poorest districts in South Africa. In addition, the prevalence of HIV in antenatal attendees within the area was estimated to be $29 \%$ in $2012 .{ }^{13}$ Although there is a paucity of data regarding the prevalence of congenital CMV in this region, the prevalence is expected to be high due to poor socio-economic conditions and the high prevalence of HIV. We therefore undertook this study to 
Table 1: Demographic characteristics

\begin{tabular}{|c|c|c|c|c|}
\hline Variable & Category & \multicolumn{2}{|c|}{ n (\%; 95\% confidence interval) } & $p$-value \\
\hline \multirow[t]{3}{*}{ Sex } & Male & \multicolumn{2}{|c|}{$146(48.34 ; 42.71-53.98)$} & \multirow[t]{3}{*}{0.416} \\
\hline & Female & $156(5$ & & \\
\hline & Total & \multicolumn{2}{|c|}{$302(100)$} & \\
\hline \multirow[t]{3}{*}{ Birth weight (g) } & $\leq 2400$ & \multicolumn{2}{|c|}{$74(24.50 ; 19.96-29.70)$} & 1 \\
\hline & $2400-3500$ & \multicolumn{2}{|c|}{$197(65.23 ; 59.65-70.42)$} & $<0.0001$ \\
\hline & $\geq 3500$ & \multicolumn{2}{|c|}{$31(10.26 ; 7.30-14.25)$} & $<0.0001$ \\
\hline \multirow[t]{2}{*}{ Mother's HIV status } & Positive & \multicolumn{2}{|c|}{$105(34.77 ; 29.58-40.35)$} & 1 \\
\hline & Negative & \multicolumn{2}{|c|}{$194(64.24 ; 58.64-69.47)$} & $<0.0001$ \\
\hline \multirow[t]{5}{*}{ Settlement } & Peri-urban & \multicolumn{2}{|c|}{$12(3.97 ; 2.26-6.89)$} & 1 \\
\hline & Rural & \multicolumn{2}{|c|}{$268(88.74 ; 84.63-91.86)$} & $<0.0001$ \\
\hline & Urban & \multicolumn{2}{|c|}{$10(3.31 ; 1.78-6.06)$} & 0.664 \\
\hline & Farm & \multicolumn{2}{|c|}{$12(3.97 ; 2.26-6.89)$} & 1 \\
\hline & Category & Interquartile range & Median & $p$-value \\
\hline \multirow[t]{3}{*}{ Birth weight (g) by Sex } & Male & $2380-3216$ & 2850 & \multirow[t]{3}{*}{0.928} \\
\hline & Female & $2470-3247.5$ & 2810 & \\
\hline & Total & $2440-3240$ & 2840 & \\
\hline \multirow[t]{3}{*}{ Length } & Male & $46-50$ & 49 & \multirow[t]{3}{*}{0.467} \\
\hline & Female & $47-50$ & 49 & \\
\hline & Total & $46-50$ & 49 & \\
\hline \multirow[t]{3}{*}{ Head circumference } & Male & $33-36$ & 34 & \multirow[t]{3}{*}{0.249} \\
\hline & Female & $32-35$ & 34 & \\
\hline & Total & $33-35$ & 34 & \\
\hline Mother's age (years) & Age & $20-32$ & 26 & - \\
\hline
\end{tabular}

determine the prevalence and demographic determinants of congenital CMV in babies born at NMAH.

\section{Materials and methods}

A cross-sectional study to determine the prevalence of congenital CMV infection among babies born at Nelson Mandela Academic Hospital was conducted from January 2016, employing consecutive sampling. Nelson Mandela Academic Hospital is a 512-bed rural central hospital, catering for one of the poorest districts in South Africa. Approximately 4500 babies are delivered at NMAH annually, with a Caesarean section rate of approximately $75 \%$.

Mother-baby pairs delivered consecutively from Monday to Friday, who gave informed consent, were included. All those who declined to participate were excluded, as were mother-baby pairs where the HIV exposure status was unknown.

Sample size was calculated using the formula: $n=Z^{2} \alpha P(100-P) / e^{2}$. Assuming a precision (e) of 2.5\%, and an alpha $\alpha=0.05$, the sample size required was 302.14,15 Maternal characteristics collected included maternal age, maternal parity, mode of delivery, maternal rapid plasma reagin (RPR) and maternal HIV status. The neonatal demographics included chronological age at testing, gender, gestational age at birth, birth weight, length at birth and head circumference at birth. The head circumference was measured within 48 hours of birth. The gestational age (in completed weeks) was determined either by obstetric estimation or by paediatric newborn examination. Neonates were classified as small for gestational age (less than the 10th percentile) or adequate for gestational age (the 10th percentile or greater) according to a standard reference curve.
Saliva swabs were collected for CMV polymerase chain reaction $(P C R)$. The saliva samples were placed in phosphate buffered saline (PBS) and stored at $-80^{\circ} \mathrm{C}$ and sent to the NHLS pathology laboratory in Port Elizabeth for analysis. Cytomegalovirus PCR was performed on the COBAS $^{\circledR}$ AmpliPrep/COBAS $^{\circledR}$ Taqman $^{\oplus}$ CMV Test (Roche, Basel, Switzerland), which was previously validated in-house for use with saliva samples. A positive result confirmed the diagnosis of congenital CMV.

All data collected were captured and coded in Microsoft Excel ${ }^{\oplus}$ 2010 (Microsoft Corp, Redmond, WA, USA) and exported for analysis onto STATA ${ }^{\oplus} 14.1$ (Stata Corp LP, College Station, TX, USA). Binomial logistic regression for estimation of risk/ prevalence ratio was used to determine the predictors of congenital CMV. Only bivariate associations are presented since model building did not yield a model that is better than the constant.

\section{Results}

A total of 307 mothers were approached to be included in the study. Three were excluded due to unknown maternal HIV status at the time of delivery, while two mothers did not provide informed consent and were thus excluded. A total of 302 births were assessed (Table 1). Almost two-thirds of births (64.24\%; 95\% Cl 58.64-69.47) were between 2400 and $3500 \mathrm{~g}$ at birth. Some $50 \%$ of the mothers were at least 26 years old and only $25 \%$ of the mothers gave birth after the age of 32 years. Over a third of the babies were HIV exposed as 105 mothers (34.77\%; 95\% Cl 29.58-40.35) were HIV positive at delivery. All the HIVexposed newborns had a negative PCR when they were tested within three days of birth. 
Table 2: Demographic predictors of congenital CMV at NMAH

\begin{tabular}{|l|c|c|c|}
\hline \multirow{2}{*}{ Variable } & Category & $\begin{array}{c}\text { PR (95\% confidence } \\
\text { interval) }\end{array}$ & p-value \\
\hline Baby's sex & Male & 1 & 1 \\
\hline & Female & $1.00(0.95-1.06)$ & 0.885 \\
\hline Birth weight (g) & $\leq 2400$ & 1 & 1 \\
\hline & $2400-3500$ & $1.05(0.97-1.14)$ & 0.249 \\
\hline Mother's age (years) & $\geq 3500$ & $1.07(0.97-1.18)$ & 0.182 \\
\hline & $\leq 19$ & 1 & 1 \\
\hline Mother's parity & $20-32$ & $1.06(0.96-1.16)$ & 0.237 \\
\hline & $33-46$ & $1.07(0.96-1.18)$ & 0.215 \\
\hline & 1 & 1 & 1 \\
\hline & 2 & $1.05(0.97-1.14)$ & 0.206 \\
\hline & 3 & $1.02(0.94-1.11)$ & 0.626 \\
\hline & $\geq 4$ & $1.06(0.99(1.14)$ & 0.084 \\
\hline Mother's HIV status & Positive & 1 & 1 \\
\hline & Negative & $1.00(0.94-1.06)$ & 0.869 \\
\hline
\end{tabular}

Congenital CMV was prevalent in 18 births $(5.96 \%$; $95 \% \mathrm{Cl} 3.29-$ 8.63) and had an equal prevalence between HIV-exposed and HIV-unexposed newborns (prevalence ratio $[\mathrm{PR}]=1.00 ; 95 \% \mathrm{Cl}$ $0.94-1.06 ; p=0.869$ ). Children with a birth weight of $2400 \mathrm{~g}$ or less were either $5 \%$ or $7 \%$ more likely to have congenital CMV than those with birth weights of between 2400 and $3500 \mathrm{~g}$ (PR $=1.05 ; 95 \% \mathrm{Cl} 0.97-1.14 ; p=0.249)$ and $3500 \mathrm{~g}$ or more $(\mathrm{PR}=$ $1.07 ; 95 \% \mathrm{Cl} 0.97-1.18 ; p=0.182$ ) respectively. These prevalence ratios (Table 2), however, were not statistically significant. Firstborn babies had a 5\%, $2 \%$ and $6 \%$ higher risk of being born with congenital CMV than babies born to mothers with parities of 2, 3 and 4 or more respectively. Congenital CMV infection was not significantly associated with the economic status or other maternal demographic characteristics (Table 2).

\section{Discussion}

This was the first study seeking to estimate the prevalence of congenital CMV at NMAH in Mthatha in the Eastern Cape province of South Africa. To date most prevalence studies on congenital CMV are from resource-rich countries whereas there is a paucity of data in resource-limited countries like South Africa. In 2014, Manicklal et al. evaluated the prevalence of congenital CMV in HIV-exposed infants in the Western Cape, South Africa, and found the prevalence of congenital CMV amongst this cohort to be $2.9 \%$. They showed that type and length of ARV prophylaxis was not significantly different between CMV infected and uninfected infants. Studies of congenital CMV from other resource-limited settings show a prevalence ranging from $1 \%$ to $5 \%, 2,14,16$ while the prevalence ranges from $0.6 \%$ to $0.7 \%$ in resource-rich countries, where maternal seroimmunity is lower. ${ }^{2}$ In addition, HIV-infected newborns are more likely to acquire congenital CMV than HIV-uninfected neonates, ${ }^{11}$ whilst there have been conflicting reports on whether or not the incidence of vertical transmission of CMV in HIV-positive mothers is falling in the ARV era. ${ }^{11,12}$ The main finding of this study is a prevalence of congenital CMV of $5.96 \%(95 \% \mathrm{Cl} 3.29-8.63)$ among study participants, which is similar to findings from other resourcelimited settings. ${ }^{14,16}$ There was no significant association between maternal HIV status and congenital CMV, as the prevalence of the latter was equal in HIV-exposed versus HIV-unexposed neonates. Previous studies have shown that a maternal CD4 count $<200$ cells $/ \mu \mathrm{L}$ during pregnancy was independently associated with congenital CMV. ${ }^{2}$ Evaluation of CD4 count was beyond the scope of this study, but it is possible that with the extensive roll-out of CART in South Africa, where all pregnant women qualify for HIV treatment irrespective of CD4 count, the majority of participants were well controlled on treatment, thus explaining the lack of a significant association between maternal HIV status and congenital CMV. In addition, none of the demographic characteristics listed in Table 2 were significantly associated with congenital CMV.

One of the limitations of this study was the relatively small but acceptable sample size, ${ }^{15}$ which could have affected the final prevalence result. In addition, it was beyond the scope of this study to evaluate the prevalence of symptomatic versus asymptomatic congenital CMV, as this would require not only physical examination of the newborns looking for the classical symptoms of congenital infections such as petechiae, hepatosplenomegaly and microcephaly, amongst others, but also clinical pathology testing to ascertain abnormalities such as thrombocytopenia and hyperbilirubinemia, as well as imaging such as head ultrasonography to screen for intracranial calcifications, for which resources were not available. This would be important to elucidate in future studies, as it has a direct impact on the health system, and may provide information that could affect how clinicians handle cases or suspected cases of congenital CMV, especially with regard to screening or diagnosis.

In conclusion, this study provides important data regarding the prevalence of congenital CMV in our setting and forms the basis for future studies on this potentially severe disease. With the prevalence of congenital CMV being as high as it is, clinicians are advised to have a high index of suspicion, especially when mothers are CMV seropositive.

Disclosure statement - No potential conflict of interest was reported by the authors.

Ethical clearance - Ethical clearance was obtained from the Human Research Ethics Committee of Walter Sisulu University (protocol number 029/2017).

Acknowledgements - The authors would like to thank the management of Nelson Mandela Academic Hospital who gave them all the necessary support. They would also like to thank the National Health Laboratory Service for the use of their facilities and resources. The authors thank the NHLS Research Trust for granting them funding to perform the laboratory testing. Most of all, the study would not have been possible without the support of patients who provided consent to participate in the study without any complaints or expectation of rewards. The authors thank you.

Funding - This work was supported by the NHLS Research Trust (grant number GRANT004_94534).

\section{ORCID}

Sikhumbuzo A Mabunda (D) http://orcid.org/0000-0001-94583742

\section{References}

1. Nelson CT, Demmler GJ. Cytomegalovirus infection in the pregnant mother, fetus, and newborn infant. Clin Perinatol. 1997;24(1): 151-60.

2. Manicklal S, van Niekerk AM, Kroon SM, et al. Birth prevalence of congenital cytomegalovirus among infants of HIV-infected women on prenatal antiretroviral prophylaxis in South Africa. Clin Infect Dis. 
2014;58(10): 1467-72. doi:10.1093/cid/ciu096. Epub 2014 Feb 23.

3. Pass RF, Hutto $C$, Ricks $R$, Cloud GA. Increased rate of cytomegalovirus infection among parents of children attending day-care centers. N Engl J Med. 1986;314(22): 1414-18. https://doi.org/10.1056/NEJM198605293142204

4. Kenneson A, Cannon MJ. Review and meta-analysis of the epidemiology of congenital cytomegalovirus (CMV) infection. Rev Med Virol. 2007;17(4): 253-76. https://doi.org/10.1002/(ISSN)1099-1654

5. Picone O, Vauloup-Fellous C, Cordier AG, et al. A series of 238 cytomegalovirus primary infections during pregnancy: description and outcome. Prenat Diagn. 2013;33(8): 751-8. doi:10.1002/pd.4118. Epub 2013 May 1.

6. Goderis J, De Leenheer E, Smets K, et al. Hearing loss and congenital CMV infection: a systematic review. Pediatrics. 2014;134(5): 972-82. doi:10.1542/peds.2014-1173.

7. Williamson WD, Demmler GJ, Percy AK, Catlin FI. Progressive hearing loss in infants with asymptomatic congenital cytomegalovirus infection. Pediatrics. 1992;90: 862-866.

8. Fowler KB, McCollister FP, Dahle AJ, Boppana S, Britt WJ, Pass RF. Progressive and fluctuating sensorineural hearing loss in children with asymptomatic congenital cytomegalovirus infection. J Pediatr. 1997(4);130: 624-30. https://doi.org/10.1016/S0022-3476(97)70248-8

9. Boppana SB, Ross SA, Fowler KB. Congenital cytomegalovirus infection: clinical outcome. Clin Infect Dis. 2013;57(Suppl 4): S178S181. doi:10.1093/cid/cit629.

10. Manicklal S, Emery VC, Lazzarotto T, Boppana SB, Gupta RK. The "silent" global burden of congenital cytomegalovirus. Clin Microbiol
Rev. 2013;26(1): 86-102. doi:10.1128/CMR.00062-12.

11. Guibert G, Warszawski J, Le Chenadec J, et al. Decreased risk of congenital cytomegalovirus infection in children born to HIV-1-infected mothers in the era of highly active antiretroviral therapy. Clin Infect Dis. 2009(11);48: 1516-25. https://doi.org/10.1086/598934

12. Frederick $T$, Homans J, Spencer $L$, et al. The effect of prenatal highly active antiretroviral therapy on the transmission of congenital and perinatal/early postnatal cytomegalovirus among HIV-infected and HIV-exposed infants. Clin Infect Dis. 2012;55(6): 877-84. doi:10.1093/cid/cis535. Epub 2012 Jun 6.

13. Shisana O, Rehle T, Simbayi LC, et al. South African National HIV Prevalence, Incidence and Behaviour Survey, 2012. Cape Town: HSRC Press; 2014.

14. van der Sande MA, Kaye S, Miles DJ, et al. Risk factors for and clinical outcome of congenital cytomegalovirus infection in a peri-urban West-African birth cohort. PLoS One 2007;2(6): e492. https://doi.org/10.1371/journal.pone.0000492

15. Naing L, Winn T, Rusli BN. Practical Issues in Calculating the Sample Size for Prevalence Studies. Archives of Orofacial Sciences. 2006;1: 9-14.

16. Dar L, Pati SK, Patro AR, et al. Congenital cytomegalovirus infection in a highly seropositive semi-urban population in India. Pediatr Infect Dis J. 2008;27(9): 841-3. https://doi.org/10.1097/INF.0b013e3181723d55

Received: 21-09-2017 Accepted: 05-04-2018 